\title{
Constant length substitutions, iterated function systems and amorphic complexity
}

\author{
Gabriel Fuhrmann ${ }^{1} \cdot$ Maik Gröger ${ }^{2}$
}

Received: 8 April 2019 / Accepted: 10 August 2019 / Published online: 22 November 2019

(c) The Author(s) 2019

\begin{abstract}
We show how geometric methods from the general theory of fractal dimensions and iterated function systems can be deployed to study symbolic dynamics in the zero entropy regime. More precisely, we establish a dimensional characterization of the topological notion of amorphic complexity. For subshifts with discrete spectrum associated to constant length substitutions, this characterization allows us to derive bounds for the amorphic complexity by interpreting the subshift as the attractor of an iterated function system in a suitable quotient space. As a result, we obtain the general finiteness and positivity of amorphic complexity in this setting and provide a closed formula in case of a binary alphabet.
\end{abstract}

\section{Introduction}

The relation between the dimension theory of dynamical systems and ergodic theory is nowadays a well-established field of research. One of the first to formally show this relation explicitly was Billingsley [4]. He proved that for an expanding circle map $f: x \mapsto b x \bmod 1$ with an $f$-invariant measure $\mu$ the following equality holds

$$
\operatorname{dim}_{H}(\mu)=\frac{h_{\mu}(f)}{\log b},
$$

where $\operatorname{dim}_{H}(\mu)$ denotes the Hausdorff dimension of the measure $\mu$ and $h_{\mu}(f)$ the measure theoretic entropy of $f$ with respect to $\mu$. Later, Furstenberg [18] established a topological analogue of this equality, namely, $\operatorname{dim}_{H}(K)=h_{\text {top }}\left(\left.f\right|_{K}\right) / \log b$ for any $f$-invariant set $K$

\footnotetext{
This project has received funding from the European Union's Horizon 2020 research and innovation program under the Marie Skłodowska-Curie grant agreement No 750865. Further, MG acknowledges support by the DFG grants JA 1721/2-1 and GR 4899/1-1 and would like to thank Henna Koivusalo for very helpful discussions concerning the theory of iterated function systems on general metric spaces.

$\bowtie$ Maik Gröger

maik.groeger@univie.ac.at

Gabriel Fuhrmann

gabriel.fuhrmann@imperial.ac.uk

1 Department of Mathematics, Imperial College London, 180 Queen's Gate, London SW7 2AZ, UK

2 Faculty of Mathematics, University of Vienna, Oskar Morgensternplatz 1, 1090 Vienna, Austria
} 
in the circle with $\operatorname{dim}_{H}(K)$ denoting the Hausdorff dimension of the set $K$ and $h_{\text {top }}\left(\left.f\right|_{K}\right)$ the topological entropy of $f$ restricted to $K$.

The relevance of these equalities originates from the fact that they relate different concepts measuring the "size" of an invariant object. Roughly speaking, entropy determines the size by measuring the amount of the supported disorder and Hausdorff dimension specifies an actually geometric size. In the concrete setting described above, there is an intermediary needed to bring both notions together, represented here by the term $\log b$. It also allows for a dynamical interpretation, namely as the Lyapunov exponent of $f$ (see, for instance, [3] for more information regarding this notion). In this article we will be mainly concerned with systems in the symbolic setting and there, no intermediary is needed as we explain in the following paragraph.

Furstenberg's result actually holds in the general context of symbolic dynamical systems. Suppose we are given a subshift $(X, \sigma)$ of $\left(A^{\mathbb{Z}}, \sigma\right)$ where $A$ is a finite alphabet, $\sigma$ is the left shift and $A^{\mathbb{Z}}$ is equipped with the Cantor metric $d_{\beta}(x, y)=\beta^{-j}$ with $\beta>1$ and $j=\min \left\{|k|: x_{k} \neq y_{k}\right\}$ for $x=\left(x_{k}\right)_{k \in \mathbb{Z}}$ and $y=\left(y_{k}\right)_{k \in \mathbb{Z}}$ in $A^{\mathbb{Z}}$. Then Furstenberg's result reads as follows (see also [34] for a generalization to higher-dimensional subshifts)

$$
\operatorname{dim}_{H}(X)=\frac{h_{\text {top }}\left(\left.\sigma\right|_{X}\right)}{\log \beta} .
$$

Moreover, for the box dimension $\operatorname{dim}_{B}$ of $X$ (see Sect. 6) it is easily seen that

$$
\operatorname{dim}_{B}(X)=\frac{h_{\mathrm{top}}\left(\left.\sigma\right|_{X}\right)}{\log \beta} .
$$

Summing up, for symbolic systems, the correspondence between entropy and dimension can be established directly. Here, the term $\log \beta$ is just a normalizing constant, depending on the chosen metric on $X$, and we can regard (topological) entropy and (Hausdorff and box) dimension in the symbolic setting as one and the same quantity.

For subshifts of zero entropy, equations (1.1) and (1.2) do not yield much insight. However, there are many natural and interesting families of symbolic systems with zero entropy. For instance, Sturmian and regular Toeplitz subshifts belong to this class. Another important subclass are substitutive subshifts including those related to the Pisot Conjecture. For more information concerning these systems, see for example [13,25] and [32]. Therefore, a desirable goal for symbolic dynamics in this low complexity regime is to find an analogously intertwined behavior of (slow entropy) concepts measuring disorder on the one side and suitable fractal dimensions on the other side.

In this article we provide this kind of relation for the topological notion of amorphic complexity which was recently introduced in [17] to study dynamical systems with zero entropy. More precisely, we show that amorphic complexity of symbolic systems coincides with the box dimension of an associated subset in the so-called Besicovitch space of $\left(A^{\mathbb{Z}}, \sigma\right)$ endowed with a canonical metric, see Lemma 6.2 and Theorem 6.5.

In fact, as we will see, this equality can be established by elementary means. At the same time, however, it embodies a key insight which puts us in a position where we can utilize methods from fractal geometry. For instance, this allows us to immediately deduce that amorphic complexity of mean equicontinuous subshifts (see Sect. 4) is bounded from below by the topological dimension of their maximal equicontinuous factor. We will explain this in more detail at the end of Sect. 6, where we also relate this observation to substitutive subshifts associated to substitutions of Pisot type.

The connection between amorphic complexity and box dimension in the symbolic setting is crucial for our main results. In particular, this relation enables us to deploy the theory 
of iterated function systems on general complete metric spaces to estimate the amorphic complexity of subshifts with discrete spectrum associated to primitive constant length substitutions, see Sect. 7. To state some of the results, let us briefly provide a background on amorphic complexity and substitutive subshifts.

For now we introduce amorphic complexity for symbolic systems. The general definition can be found in Sect. 3, where we also provide a short overview of some of the basic properties of this topological invariant. Given a subshift $(X, \sigma), x, y \in X$ and $\delta>0$, we set

$$
D_{\delta}(x, y)=\varlimsup_{n \rightarrow \infty} \frac{\#\left\{0 \leq k \leq n-1: d\left(\sigma^{k}(x), \sigma^{k}(y)\right) \geq \delta\right\}}{n},
$$

where $d$ can be any metric generating the product topology on $A^{\mathbb{Z}}$. We define the asymptotic separation numbers as

$$
\operatorname{Sep}\left(\left.\sigma\right|_{X}, \delta, v\right)=\sup \left\{\# Y: Y \subseteq X \text { with } D_{\delta}(x, y) \geq v \text { for all } x \neq y \text { in } Y\right\},
$$

with $\delta>0$ and $v \in(0,1]$. The amorphic complexity of $(X, \sigma)$ is set to be

$$
\operatorname{ac}\left(\left.\sigma\right|_{X}\right)=\sup _{\delta>0} \lim _{\nu \rightarrow 0} \frac{\log \operatorname{Sep}\left(\left.\sigma\right|_{X}, \delta, v\right)}{-\log v},
$$

whenever the limit in $v$ exists (otherwise, we define the lower and upper amorphic complexity $\underline{\mathrm{ac}}$ and $\overline{\mathrm{ac}}$, by taking the limit inferior and limit superior, respectively).

A substitution over a finite alphabet $\mathrm{A}$ is a map $\vartheta: A \rightarrow A^{+}$, where $A^{+}$is the set of all non-empty finite words with letters from $A$. Observe that by concatenation, $\vartheta$ can be considered a map from $A^{+}$to $A^{+}$and from $A^{\mathbb{Z}}$ to $A^{\mathbb{Z}}$ in the natural way. We call a substitution $\vartheta$ primitive if for some $\ell>0$ and every $a \in A$ the word $\vartheta^{\ell}(a)$ contains each of the letters of $A$. We say a substitution $\vartheta$ is of constant length if there is $\ell \in \mathbb{N}$ such that $\vartheta(a) \in A^{\ell}$ for each $a \in A$ (and we set $|\vartheta|=\ell$ ). For every constant length substitution $\vartheta$ there exists a periodic point $x_{0}$ of $\vartheta$ in $A^{\mathbb{Z}}$, that is, there is $p \in \mathbb{N}$ with $\vartheta^{p}\left(x_{0}\right)=x_{0}$ (see [20]). Finally, if $\vartheta$ is primitive, we denote by $X_{\vartheta}$ the shift orbit closure of some periodic point of $\vartheta$. This notation is justified because $X_{\vartheta}$ turns out to be independent of the particular $\vartheta$-periodic point. We call $\left(X_{\vartheta}, \sigma\right)$ a substitution (or substitutive) subshift. It is well known that $X_{\vartheta}$ is minimal [20] and has a unique Borel probability measure which is invariant under the action of the left shift [27].

Let us now state our main results. We start by considering substitutions over two symbols whose associated substitutive subshift is infinite (a subshift $(X, \sigma)$ is called finite if $X$ is finite; otherwise it is called infinite). In this case, we can establish a closed formula for the amorphic complexity of $\left(X_{\vartheta}, \sigma\right)$. The proof of the next statement can be found at the end of Sect. 7.2.

Theorem 1.1 Let $\vartheta:\{0,1\} \rightarrow\{0,1\}^{+}$be a primitive constant length substitution. Assuming that $\left(X_{\vartheta}, \sigma\right)$ is infinite, we get

$$
\operatorname{ac}\left(\left.\sigma\right|_{X_{\vartheta}}\right)=\frac{\log |\vartheta|}{\log |\vartheta|-\log |\vartheta|_{*}},
$$

with $0<|\vartheta|_{*} \leq|\vartheta|$ the number of positions where $\vartheta(0)$ and $\vartheta(1)$ differ.

An interesting consequence of Theorem 1.1 is that the amorphic complexity of infinite substitution subshifts over two symbols with discrete spectrum is always finite and bounded from below by one. As it turns out, this holds true over general alphabets. 
Theorem 1.2 Assume $\vartheta: A \rightarrow A^{+}$is a primitive substitution of constant length. If $\left(X_{\vartheta}, \sigma\right)$ is infinite and has discrete spectrum, then

$$
1 \leq \underline{\operatorname{ac}}\left(\left.\sigma\right|_{X_{\vartheta}}\right) \leq \overline{\mathrm{ac}}\left(\left.\sigma\right|_{X_{\vartheta}}\right)<\infty .
$$

We want to point out that the proof of the previous theorem yields means to compute concrete lower and upper bounds for the amorphic complexity of infinite substitutive subshifts with discrete spectrum, see Sect. 7.3. Finally, using Theorem 1.2 and further properties of substitution subshifts together with the general theory of amorphic complexity, we obtain the following trichotomy, see also Sect. 7.3.

Corollary 1.3 Suppose $\vartheta: A \rightarrow A^{+}$is a primitive substitution of constant length.

(i) $\operatorname{ac}\left(\left.\sigma\right|_{X_{\vartheta}}\right)=0$ iff $\left(X_{\vartheta}, \sigma\right)$ is finite.

(ii) $1 \leq \underline{\operatorname{ac}}\left(\left.\sigma\right|_{X_{\vartheta}}\right) \leq \overline{\mathrm{ac}}\left(\left.\sigma\right|_{X_{\vartheta}}\right)<\infty$ iff $\left(X_{\vartheta}, \sigma\right)$ has discrete spectrum and is infinite.

(iii) $\operatorname{ac}\left(\left.\sigma\right|_{X_{\vartheta}}\right)=\infty$ iff $\left(X_{\vartheta}, \sigma\right)$ has partly continuous spectrum.

\section{Basic notation and definitions}

Given a continuous self-map $f: X \rightarrow X$ on a compact metric space $(X, d)$, we call the pair $(X, f)$ a (topological) dynamical system. We say $(X, f)$ is invertible if $f$ is invertible. ${ }^{1}$ Given two dynamical systems $(X, f)$ and $(Y, g)$, we say $(Y, g)$ is a factor of $(X, f)$ if there exists a continuous onto map $h: X \rightarrow Y$ such that $h \circ f=g \circ h$. If additionally, $h$ is invertible, then $h$ is a conjugacy and we say $(X, f)$ and $(Y, g)$ are conjugate.

A system $(X, f)$ is equicontinuous if the family $\left(f^{n}\right)_{n \in \mathbb{N}}$ is uniformly equicontinuous, that is, if for all $v>0$ there is $\delta>0$ such that for all $x, y \in X$ with $d(x, y)<\delta$ we have $d\left(f^{n}(x), f^{n}(y)\right)<v(n \in \mathbb{N})$. It is well known, see for instance [9, Theorem 2.1], that every dynamical system $(X, f)$ has a unique (up to conjugacy) maximal equicontinuous factor: an equicontinuous factor $(Y, g)$ so that every other equicontinuous factor of $(X, f)$ is also a factor of $(Y, g)$.

A subset $E \subseteq X$ is $f$-invariant if it is closed and $f(E)=E$. In this case, we call $\left(E,\left.f\right|_{E}\right)$ (and sometimes synonymously $E$ itself) a subsystem of $(X, f)$ and usually just write $(E, f)$ for notational convenience. We say $E$ is $f$-minimal if it is $f$-invariant and does not contain any non-empty proper subset which is $f$-invariant. In case that $X$ is $f$-minimal itself, we also say that $(X, f)$ is minimal.

Given a topological dynamical system $(X, f)$, a Borel probability measure $\mu$ on $X$ is called $f$-invariant if $\mu\left(f^{-1}(E)\right)=\mu(E)$ for all Borel measurable sets $E \subseteq X$. An invariant measure $\mu$ is called ergodic if for all Borel measurable $E \subseteq X$ with $f^{-1}(E)=E$ we have $\mu(E) \in\{0,1\}$. We say $(X, f)$ is uniquely ergodic if there exists exactly one $f$-invariant measure $\mu$. Note that in this case, the unique invariant measure $\mu$ is ergodic.

We will mainly deal with bi-infinite shift spaces $\Sigma=A^{\mathbb{Z}}$, where $A$ is a finite set also referred to as alphabet and $\Sigma$ carries the product topology. We define the full shift to be the system $(\Sigma, \sigma)$, where $\sigma \Sigma \rightarrow \Sigma$ is the left shift, that is, $\sigma\left(\left(x_{n}\right)_{n \in \mathbb{Z}}\right)=\left(x_{n+1}\right)_{n \in \mathbb{Z}}$ for all $\left(x_{n}\right)_{n \in \mathbb{Z}} \in \Sigma$. For $n \in \mathbb{N}$ elements of $A^{n}$ are called words of length $n$. We set $A^{+}=\bigcup_{n \in \mathbb{N}} A^{n}$.

Subsystems of the full shift are referred to as subshifts. One way to obtain subshifts is to consider orbit closures: given $x_{0} \in \Sigma$, we define its orbit closure to be $X_{x_{0}}=$

1 For future reference, we would like to phrase some of the preliminary results in the non-invertible setting, although our main results are actually concerned with invertible systems. 
$\overline{\left\{\sigma^{k}\left(x_{0}\right): k \in \mathbb{Z}\right\}} \subseteq \Sigma$. Clearly, $X_{x_{0}}$ is $\sigma$-invariant. Given a subshift $(X, \sigma)$, the collection of all words of length $n$ that appear in $X$ is denoted by $\mathcal{L}^{n}(X)$. That is, $w \in \mathcal{L}^{n}(X)$ if and only if $w \in A^{n}$ and there is $x \in X$ such that $w_{i}=x_{i}(i=0, \ldots, n-1)$. Given two minimal subshifts $X \neq Y$ of $\Sigma$, it is easy to see that there is $n \in \mathbb{N}$ with $\mathcal{L}^{n}(X) \cap \mathcal{L}^{n}(Y)=\emptyset$.

\section{Asymptotic separation numbers and amorphic complexity}

In this section, we briefly introduce amorphic complexity which is a conjugacy invariant that is of particular relevance in the class of mean equicontinuous systems (see Sect. 4). Given a dynamical system $(X, f), x, y \in X$ and $\delta>0$, we set

$$
\Delta(f, \delta, x, y)=\left\{k \in \mathbb{N}_{0}: d\left(f^{k}(x), f^{k}(y)\right) \geq \delta\right\} .
$$

For $v \in(0,1]$ we say $x$ and $y$ are $(f, \delta, v)$-separated if $\bar{D}(\Delta(f, \delta, x, y)) \geq v$, where $\bar{D}(E)$ denotes the upper density of a subset $E \subseteq \mathbb{N}_{0}$ defined as

$$
\bar{D}(E)=\varlimsup_{n \rightarrow \infty} \frac{\#(E \cap[0, n-1])}{n} .
$$

A subset $S \subseteq X$ is said to be $(f, \delta, v)$-separated if all pairs of distinct points $x, y \in S$ are $(f, \delta, v)$-separated. The (asymptotic) separation numbers of $(X, f)$, denoted by $\operatorname{Sep}(f, \delta, v)$ for $\delta>0$ and $v \in(0,1]$, are defined as the largest cardinality of an $(f, \delta, v)$-separated set contained in $X$. If $\operatorname{Sep}(f, \delta, v)$ is finite for all $\delta>0, v \in(0,1]$, we say $(X, f)$ has finite separation numbers, otherwise we say it has infinite separation numbers.

As the next theorem suggests, finite separation numbers correspond to systems of low dynamical complexity, that is, in particular to systems of zero entropy and those without non-trivial weakly mixing measures (see, e.g., [35] for definitions of these concepts).

Theorem 3.1 ([17]) If a dynamical system $(X, f)$ has positive topological entropy or is weakly mixing with respect to some invariant probability measure $\mu$ with non-trivial support, then $(X, f)$ has infinite separation numbers.

For systems with finite separation numbers, we may obtain further quantitative information by studying the scaling behavior of the separation numbers as the separation frequency $v$ goes to zero. We define the lower and upper amorphic complexity of $(X, f)$ as

$$
\underline{\operatorname{ac}}(f)=\sup _{\delta>0} \varliminf_{v \rightarrow 0} \frac{\log \operatorname{Sep}(f, \delta, v)}{-\log v} \text { and } \overline{\operatorname{ac}}(f)=\sup _{\delta>0} \varlimsup_{v \rightarrow 0} \frac{\log \operatorname{Sep}(f, \delta, v)}{-\log v} .
$$

If both values coincide, we call $\operatorname{ac}(f)=\underline{\operatorname{ac}}(f)=\overline{\operatorname{ac}}(f)$ the amorphic complexity of $(X, f)$. Observe that by allowing the above quantities to assume values in $[0, \infty]$, they are actually well defined for any map $f: X \rightarrow X$. In particular, systems with infinite separation numbers have infinite amorphic complexity.

We refer the reader to [17] for an in-depth discussion of amorphic complexity and several classes of examples. However, the following statement is worth recalling.

Theorem 3.2 ([17]) Let $(X, f)$ and $(Y, g)$ be dynamical systems.

(i) Suppose $(Y, g)$ is a factor of $(X, f)$. Then $\overline{\mathrm{ac}}(f) \geq \overline{\mathrm{ac}}(g)$ and $\underline{\operatorname{ac}}(f) \geq \operatorname{ac}(g)$. In particular, amorphic complexity is an invariant of topological conjugacy.

(ii) Let $m \in \mathbb{N}$. Then $\overline{\mathrm{ac}}\left(f^{m}\right)=\overline{\mathrm{ac}}(f)$ and $\underline{\mathrm{ac}}\left(f^{m}\right)=\underline{\mathrm{ac}}(f)$. 
Since our main results involve invertible dynamical systems, let us close this section with a comment on the following issue. If $f$ is invertible, it may seem natural to define asymptotic separation numbers and amorphic complexity in a slightly different way: for $\delta>0, v \in(0,1]$ and $x, y \in X$ we could consider

$$
\Delta^{\prime}(f, \delta, x, y)=\left\{k \in \mathbb{Z}: d\left(f^{k}(x), f^{k}(y)\right) \geq \delta\right\}
$$

instead of $\Delta(f, \delta, x, y)$. Thus, we may consider a modified upper density for subsets $E \in \mathbb{Z}$,

$$
\bar{D}^{\prime}(E)=\varlimsup_{n \rightarrow \infty} \frac{\#(E \cap[-n+1, n-1])}{2 n-1},
$$

to define the $(f, \delta, v)$-separation of two points $x$ and $y$ and hence the asymptotic separation numbers as well as the amorphic complexity (where all subsequent definitions from this section would carry over in the obvious way). However, in [15] it is shown that for a natural class of invertible systems (which includes mean equicontinuous systems) this neither affects whether $(X, f)$ has infinite separation numbers nor the value of the (lower and upper) amorphic complexity. For that reason, we stick with the above definitions.

\section{Mean equicontinuity and finite separation numbers}

In this section, we discuss a canonical class of systems with finite separation numbers which in particular comprises all substitutive subshifts associated to primitive substitutions with discrete spectrum (see Sect. 5). We say a dynamical system $(X, f)$ is (Besicovitch-) mean equicontinuous if for every $v>0$ there is $\delta>0$ such that for all $x, y \in X$ with $d(x, y)<\delta$ we have

$$
D_{B}(x, y)=\varlimsup_{n \rightarrow \infty} \frac{1}{n} \sum_{k=0}^{n-1} d\left(f^{k}(x), f^{k}(y)\right)<v .
$$

This notion was introduced by $\mathrm{Li}$ et al. in [28]. It is immediately seen to be equivalent to the concept of mean Lyapunov-stability which was introduced in 1951 by Fomin [14] in the context of systems with discrete spectrum. A first systematic treatment is due to Auslander [1]. For recent activities related to these notions, see for example [10,12,16,19,23,31].

It is worth noting that $D_{B}$ is a pseudometric. With this in mind, it is not hard to see that $(X, f)$ is mean equicontinuous if and only if $D_{B} X \times X \rightarrow[0, \infty)$ is continuous. Further, it is immediate that

$$
\delta \cdot \bar{D}(\Delta(f, \delta, x, y)) \leq D_{B}(x, y) \quad(x, y \in X),
$$

where $\delta>0$. Since $X$ is compact, this yields the next statement.

Proposition 4.1 If $(X, f)$ is mean equicontinuous, then it has finite separation numbers.

In the minimal case we can say even more. To that end, let us introduce the following notion: a system $(X, f)$ is called mean sensitive if there is $v>0$ such that for every $x \in X$ and $\delta>0$ there exists $y \in X$ with $d(x, y)<\delta$ and $D_{B}(x, y)>v$.

Proposition 4.2 ([28, Corollary 5.5 \& Proposition 5.1(3)]) A minimal dynamical system $(X, f)$ is eithermean equicontinuous or mean sensitive. Moreover, if $(X, f)$ is mean sensitive, then $(X, f)$ has infinite separation numbers. 
As an immediate corollary of Proposition 4.1 and Proposition 4.2, we obtain the following assertion.

Theorem 4.3 Suppose $(X, f)$ is minimal. Then $(X, f)$ is mean equicontinuous if and only if $(X, f)$ has finite separation numbers.

Let us briefly come back to the discussion at the end of the previous section. If $f$ is invertible, we may consider the pseudometric

$$
D_{B}^{\prime}(x, y)=\varlimsup_{n \rightarrow \infty} \frac{1}{2 n-1} \sum_{k=n-1}^{n-1} d\left(f^{k}(x), f^{k}(y)\right) \quad(x, y \in X),
$$

instead of $D_{B}$. However, the results of [10] and [16,31] (the former treats the minimal case and the latter treat the general case) yield that $D_{B}$ is continuous if and only if $D_{B}^{\prime}$ is continuous. We may hence stick with $D_{B}$ in the following.

\section{Discrete spectrum and mean equicontinuity}

Suppose $(X, f)$ is a dynamical system and let $\mu$ be an $f$-invariant measure. We call $\lambda \in \mathbb{C}$ an eigenvalue and $\varphi \in L_{2}(X, \mu)$ a corresponding eigenfunction of $(X, f)$ if $\varphi \circ f=\lambda \cdot \varphi$. Further, $(X, f)$ is said to have (purely) discrete spectrum with respect to $\mu$ if there is an orthonormal basis for $L_{2}(X, \mu)$ consisting of eigenfunctions.

Theorem 5.1 ([16, Corollary 6.3]) Assume $(X, f)$ is an invertible minimal dynamical system. Then $(X, f)$ is mean equicontinuous if and only if it is uniquely ergodic, has discrete spectrum and each eigenvalue possesses a continuous eigenfunction.

For subshifts associated to primitive substitutions, a classical result by Host [22] (see [32, Theorem 6.3 \& Remark 6.3(1)], too) states that all eigenvalues possess a continuous eigenfunction. Hence, the previous theorem immediately yields the following corollary, where we make use of Theorem 4.3 and the fact that the subshift $\left(X_{\vartheta}, \sigma\right)$ associated to a primitive substitution $\vartheta: A \rightarrow A^{+}$is always minimal and uniquely ergodic.

Corollary 5.2 Let $\vartheta$ be a primitive substitution and $\left(X_{\vartheta}, \sigma\right)$ the associated subshift. Then the following are equivalent.

(i) $\left(X_{\vartheta}, \sigma\right)$ is mean equicontinuous.

(ii) $\left(X_{\vartheta}, \sigma\right)$ has discrete spectrum.

(iii) $\left(X_{\vartheta}, \sigma\right)$ has finite separation numbers.

\section{Box dimension and amorphic complexity}

In this section, we establish a connection between the amorphic complexity of a subshift and the box dimension of an associated subset in the so-called Besicovitch space.

We start by briefly introducing the box dimension of a totally bounded subset $E$ of a general metric space $(M, \rho)$. We call a subset $S$ of $M \varepsilon$-separated if for all $s \neq s^{\prime} \in S$ we have $\rho\left(s, s^{\prime}\right) \geq \varepsilon$ and denote by $M_{\varepsilon}(E)$ the maximal cardinality of an $\varepsilon$-separated subset of $E$. Then the lower and upper box dimension of $E$ are defined as

$$
\underline{\operatorname{dim}}_{B}(E)=\varliminf_{\varepsilon \rightarrow 0} \frac{\log M_{\varepsilon}(E)}{-\log \varepsilon} \quad \text { and } \quad \overline{\operatorname{dim}}_{B}(E)=\varlimsup_{\varepsilon \rightarrow 0} \frac{\log M_{\varepsilon}(E)}{-\log \varepsilon} .
$$


In case that $\underline{\operatorname{dim}}_{B}(E)$ and $\overline{\operatorname{dim}}_{B}(E)$ coincide, their common value, denoted by $\operatorname{dim}_{B}(E)$, is called the box dimension ${ }^{2}$ of $E$. We will make use of the following basic facts.

Theorem 6.1 (i) The lower and upper box dimension are invariant with respect to Lipschitzcontinuous homeomorphisms with a Lipschitz-continuous inverse.

(ii) Given a metric space $(M, \rho)$, a totallybounded subset $E \subseteq M$ and a Lipschitz-continuous map $h: E \rightarrow M$, we have

$$
\underline{\operatorname{dim}}_{B}(E)=\underline{\operatorname{dim}}_{B}\left(\bigcup_{i=0}^{n-1} h^{i}(E)\right) \leq \overline{\operatorname{dim}}_{B}\left(\bigcup_{i=0}^{n-1} h^{i}(E)\right)=\overline{\operatorname{dim}}_{B}(E) .
$$

Proof We only show the first equality in part (ii). The second equality in (ii) as well as part (i) can be shown analogously, see also [30, Theorem $6.3 \&$ Appendix I].

It is straightforward to see that

$$
\max _{0 \leq i<n} \underline{\operatorname{dim}}_{B}\left(E_{i}\right) \leq \underline{\operatorname{dim}}_{B}\left(\bigcup_{i=0}^{n-1} E_{i}\right)
$$

for totally bounded $E_{0}, E_{1}, \ldots, E_{n-1} \subseteq M$ (see also [30, Theorem $6.2 \&$ Appendix I]). It hence suffices to show $\underline{\operatorname{dim}}_{B}\left(\bigcup_{i=0}^{n-1} h^{i}(E)\right) \leq \underline{\operatorname{dim}}_{B}(E)$. To that end, let $L>1$ be a mutual Lipschitz constant of the maps $h, \ldots, h^{n-1}$. We clearly have $M_{L \varepsilon}\left(h^{i}(E)\right) \leq M_{\varepsilon}(E)$ for all $\varepsilon>0$ and each $i \in\{0, \ldots, n-1\}$. Hence,

$$
\underline{\operatorname{dim}}_{B}\left(\bigcup_{i=0}^{n-1} h^{i}(E)\right) \leq \varliminf_{\varepsilon \rightarrow 0} \frac{\log \sum_{i=0}^{n-1} M_{L \varepsilon}\left(h^{i}(E)\right)}{-\log L \varepsilon} \leq \varliminf_{\varepsilon \rightarrow 0} \frac{\log \left(n \cdot M_{\varepsilon}(E)\right)}{-\log L \varepsilon}=\underline{\operatorname{dim}}_{B}(E) .
$$

The first part of the above statement has an immediate but important corollary: if $\rho$ and $\rho^{\prime}$ are Lipschitz-equivalent metrics on $M$, that is, if there are $c, C>0$ such that

$$
c \cdot \rho(x, y) \leq \rho^{\prime}(x, y) \leq C \cdot \rho(x, y) \quad(x, y \in M),
$$

then the box dimension of any totally bounded subset $E$ of $M$ is independent of whether we compute $M_{\varepsilon}(E)$ with respect to $\rho$ or $\rho^{\prime}$.

Next, we aim at introducing the Besicovitch space associated to the full shift $(\Sigma, \sigma)$. Recall that the definition of the quantity $D_{\delta}$ in (1.3) implicitly depends on the particular metric $d$ we put on $\Sigma$. For a plethora of metrics, it turns out that $D_{\delta}$ is in fact a pseudometric and moreover, Lipschitz-equivalent to any other such pseudometric obtained from (1.3). For the next statement and its proof, we write $D_{\delta}^{d}$ to stress the dependence on $d$ explicitly. Given a metric $d$ on $\Sigma$, let $\delta_{0}^{d}>0$ be such that $d(x, y) \geq \delta_{0}^{d}$ whenever $x_{0} \neq y_{0}$ where $x=\left(x_{k}\right)_{k \in \mathbb{Z}}$ and $y=\left(y_{k}\right)_{k \in \mathbb{Z}}$ are from $\Sigma$.

Lemma 6.2 Suppose $d$ is an ultrametric ${ }^{3}$ which induces the product topology on $\Sigma$ and let $\delta>0$. Then $D_{\delta}^{d}$ is a pseudometric on $\Sigma$.

Further, assume $d$ and $d^{\prime}$ are two metrics which induce the product topology on $\Sigma$. Let $\delta \in\left(0, \delta_{0}^{d}\right]$ and $\delta^{\prime} \in\left(0, \delta_{0}^{d^{\prime}}\right]$. Then there are $c, C>0$ such that

$$
c \cdot D_{\delta}^{d}(x, y) \leq D_{\delta^{\prime}}^{d^{\prime}}(x, y) \leq C \cdot D_{\delta}^{d}(x, y) \quad(x, y \in \Sigma) .
$$

\footnotetext{
2 Let us remark that the standard definition of the box dimension involves the smallest number of sets with diameter strictly smaller than $\varepsilon$ needed to cover $E$. It is well known that the above definitions do not change if in (6.1), $M_{\varepsilon}(E)$ is replaced by this number (see also, for example, Proposition 1.4.6 in [11]).

3 Recall that an ultrametric $d$ fulfills the ultrametric inequality $d(x, y) \leq \max \{d(x, z), d(z, y)\}$ which in particular implies that two balls are either disjoint or one is contained in the other.
} 
Proof For the first part, we restrict to proving the triangle inequality since the other properties are trivial. Observe that for arbitrary $x, y, z \in \Sigma$ we have

$$
\begin{aligned}
& \#\left\{0 \leq k \leq n-1: d\left(\sigma^{k}(x), \sigma^{k}(y)\right) \geq \delta\right\} \\
& \quad \leq \#\left\{0 \leq k \leq n-1: d\left(\sigma^{k}(x), \sigma^{k}(z)\right) \geq \delta \text { or } d\left(\sigma^{k}(z), \sigma^{k}(y)\right) \geq \delta\right\} \\
& \quad \leq \#\left\{0 \leq k \leq n-1: d\left(\sigma^{k}(x), \sigma^{k}(z)\right) \geq \delta\right\}+\#\left\{0 \leq k \leq n-1: d\left(\sigma^{k}(z), \sigma^{k}(y)\right) \geq \delta\right\},
\end{aligned}
$$

using the ultrametric inequality in the first step. This yields that $D_{\delta}$ is a pseudometric.

For the second part, we first prove that for all $\eta, \eta^{\prime} \in\left(0, \delta_{0}^{d}\right]$ there are $c, C>0$ such that

$$
c \cdot D_{\eta^{\prime}}^{d}(x, y) \leq D_{\eta}^{d}(x, y) \leq C \cdot D_{\eta^{\prime}}^{d}(x, y) \quad(x, y \in \Sigma)
$$

where we consider $D_{\eta^{\prime}}^{d}$ and $D_{\eta}^{d}$ as defined in (1.3) regardless of whether this yields pseudometrics on $\Sigma$ or not. Without loss of generality, we may assume $\eta^{\prime}=\delta_{0}^{d}$.

Note that since $d$ induces the product topology on $\Sigma$, there exists $m_{\eta} \in \mathbb{N}$ such that $d(x, y) \geq \eta$ yields the existence of $\ell \in\left\{-m_{\eta}, \ldots, m_{\eta}\right\}$ with $x_{\ell} \neq y_{\ell}$. Hence,

$$
\begin{aligned}
\# & \left\{0 \leq k \leq n-1: d\left(\sigma^{k}(x), \sigma^{k}(y)\right) \geq \eta\right\} \leq \#\left\{0 \leq k \leq n-1: x_{\left[k-m_{\eta}, k+m_{\eta}\right]} \neq y_{\left[k-m_{\eta}, k+m_{\eta}\right]}\right\} \\
& \leq\left(2 m_{\eta}+1\right) \cdot \#\left\{-m_{\eta} \leq k \leq n-1+m_{\eta}: x_{k} \neq y_{k}\right\} \\
& \leq\left(2 m_{\eta}+1\right) \cdot \#\left\{-m_{\eta} \leq k \leq n-1+m_{\eta}: d\left(\sigma^{k}(x), \sigma^{k}(y)\right) \geq \delta_{0}^{d}\right\} .
\end{aligned}
$$

Therefore, for all $x, y \in \Sigma$ we have the right-hand side of (6.3) with $C=2 m_{\eta}+1$. Further, we trivially have $D_{\delta_{0}^{d}}^{d}(x, y) \leq D_{\eta}^{d}(x, y)$ and hence the left-hand side of (6.3) with $c=1$.

Finally, let $d, d^{\prime}, \delta$ and $\delta^{\prime}$ be as in the statement. Since $d$ and $d^{\prime}$ induce the same topology, there is $\eta \in\left(0, \delta_{0}^{d}\right]$ such that $d^{\prime}(x, y) \geq \delta^{\prime}$ implies $d(x, y) \geq \eta$. With such $\eta$, we have for all $x, y \in \Sigma$

$$
D_{\delta^{\prime}}^{d^{\prime}}(x, y) \leq D_{\eta}^{d}(x, y) \leq C \cdot D_{\delta}^{d}(x, y),
$$

for some $C>0$ which exists due to (6.3). By a similar argument, we obtain $D_{\delta}^{d}(x, y) \leq$ $1 / c \cdot D_{\delta^{\prime}}^{d^{\prime}}(x, y)$ for some $c>0$. This finishes the proof.

Following a standard procedure, we introduce an equivalence relation on $\Sigma$ by identifying $x, y \in \Sigma$ whenever $D_{\delta}(x, y)=0$ and denote by [.] the corresponding quotient mapping. Note that Lemma 6.2 ensures that this relation is actually an equivalence relation and that is well defined, i.e., independent of the particular metric $d$ on $\Sigma$ and independent of $\delta \in\left(0, \delta_{0}^{d}\right]$. Sometimes, we may consider subshifts with different alphabets. In those cases, we may use the notation $[\cdot]_{A}$ to unambiguously specify the corresponding quotient mapping (recall that $A$ denotes the alphabet of $\Sigma$, see Sect. 2).

For the rest of this work, we may assume without loss of generality that $d$ is a Cantor metric (so that $\delta_{0}^{d}=1$ ). Since $d$ is hence an ultrametric, $D_{\delta}$ is a pseudometric on $\Sigma$ for each $\delta \in(0,1]$, which we may further consider a metric on $[\Sigma]$ in the canonical way. We call $\left([\Sigma], D_{\delta}\right)$ the Besicovitch space.

Since most of the analysis carried out in this work takes place in the space ([ $\left.\Sigma], D_{\delta}\right)$, we provide the next statement to familiarize the reader with its topological and metric properties. Its proof is a straightforward application of the results in [5].

Theorem 6.3 The space $\left([\Sigma], D_{\delta}\right)$ is complete and pathwise connected. Moreover, it is topologically infinite dimensional and neither locally compact nor separable. 
Proof First, recall that it suffices to show the statement for ([ $\left.\Sigma], D_{1}\right)$, see Lemma 6.2. Analogously to the definition of $D_{1}$, we may consider

$$
D_{1}^{\prime}(x, y)=\varlimsup_{n \rightarrow \infty} \frac{\#\left\{n-1 \leq k \leq n-1: x_{k} \neq y_{k}\right\}}{2 n-1} \quad(x, y \in \Sigma) .
$$

Similarly as in Lemma 6.2, we see that $D_{1}^{\prime}$ defines a pseudometric (see also [5]) and hence an equivalence relation by identifying points $x, y \in \Sigma$ whenever $D_{1}^{\prime}(x, y)=0$. Let us denote the corresponding quotient mapping by $[\cdot]^{\prime}$ and, as before, consider $D_{1}^{\prime}$ a metric on $[\Sigma]^{\prime}$ in the canonical way.

Now, [5, Proposition 1-3] state that $\left([\Sigma]^{\prime}, D_{1}^{\prime}\right)$ is complete, pathwise connected, topologically infinite dimensional and neither locally compact nor separable. We simply carry over these properties to $\left([\Sigma], D_{1}\right)$ by means of the map

$$
\varphi:[\Sigma]^{\prime} \rightarrow[\Sigma]:\left[\left(\ldots, x_{-2}, x_{-1}, x_{0}, x_{1}, x_{2}, \ldots\right)\right]^{\prime} \mapsto\left[\left(\ldots, x_{-2}, x_{-1}, x_{0}, x_{-1}, x_{1}, x_{-2}, x_{2}, \ldots\right)\right]
$$

Note that $\varphi$ is isometric and surjective. The statement follows.

Now, observe that, given a subshift $(X, \sigma)$, we have $D_{\delta}(x, y)=\bar{D}\left(\Delta\left(\left.\sigma\right|_{X}, \delta, x, y\right)\right)$ for all $\delta \in(0,1]$ and $x, y \in X$. In other words,

$$
\operatorname{Sep}\left(\left.\sigma\right|_{X}, \delta, v\right)=\sup \left\{\# Y: Y \subseteq X \text { with } D_{\delta}(x, y) \geq v \text { for all } x \neq y \text { in } Y\right\}
$$

as stated in the introduction. The proof of the next statement is hence straightforward.

Proposition 6.4 Given a subshift $(X, \sigma)$ and $\delta \in(0,1]$. Then $\operatorname{Sep}\left(\left.\sigma\right|_{X}, \delta, v\right)$ is finite for all $v \in(0,1]$ if and only if $[X]$ is totally bounded in $\left([\Sigma], D_{\delta}\right)$.

The following theorem shows that studying the amorphic complexity of a subshift $(X, \sigma)$ amounts to studying the box dimension of $[X]$.

Theorem 6.5 Suppose $(X, \sigma)$ is a subshift with finite separation numbers. Then the (lower and upper) box dimension of the subset $[X]$ of $[\Sigma]$ (equipped with $D_{\delta}$ ) is independent of $\delta \in(0,1]$. Further,

$$
\underline{\operatorname{ac}}\left(\left.\sigma\right|_{X}\right)=\underline{\operatorname{dim}}_{B}([X]) \text { and } \overline{\operatorname{ac}}\left(\left.\sigma\right|_{X}\right)=\overline{\operatorname{dim}}_{B}([X]) \text {. }
$$

Proof Fix $\delta \in(0,1]$. Since $(X, \sigma)$ has finite separation numbers, the lower and upper box dimension of $[X]$ in $\left([\Sigma], D_{\delta}\right)$ are well defined, according to Proposition 6.4. A priori, their values may still depend on $\delta$. Yet, using the Lipschitz-equivalence of the $D_{\delta}$ 's (see Lemma 6.2) and the Lipschitz-invariance of the box dimension (see Theorem 6.1(i)), we have that the lower and upper box dimension of $[X]$ in $\left([\Sigma], D_{\delta}\right)$ are independent of $\delta$. This yields the first part of the statement.

For the second part, observe that for each $v \in(0,1]$ we have $\operatorname{Sep}\left(\left.\sigma\right|_{X}, \delta, v\right)=M_{v}^{\delta}([X])$, where $M_{v}^{\delta}([X])$ denotes the maximal cardinality of a $\nu$-separated subset of $[X]$ equipped with $D_{\delta}$. Then, 


$$
\begin{aligned}
\underline{\operatorname{ac}}\left(\left.\sigma\right|_{X}\right) & =\sup _{\delta>0} \underline{\lim }_{\nu \rightarrow 0} \frac{\log \operatorname{Sep}\left(\left.\sigma\right|_{X}, \delta, v\right)}{-\log v}=\sup _{\delta>0} \frac{\lim }{\nu \rightarrow 0} \frac{\log M_{\nu}^{\delta}([X])}{-\log v}=\sup _{\delta>0} \underline{\operatorname{dim}}_{B}([X]) \\
& =\underline{\operatorname{dim}}_{B}([X]) .
\end{aligned}
$$

Clearly, a similar statement holds for the upper amorphic complexity.

Due to the previous statement, we are now in a position to study the amorphic complexity from a geometric perspective. For instance, we can understand the topological invariance of amorphic complexity (of symbolic systems) also from the perspective of the following lemma.

Lemma 6.6 Assume that $X \subseteq A^{\mathbb{Z}}$ and $Y \subseteq B^{\mathbb{Z}}$ are $\sigma^{n}$ - and $\sigma^{m}$-invariant, respectively, with $n, m \in \mathbb{N}$. If $h$ is a factor map from $\left(X, \sigma^{n}\right)$ to $\left(Y, \sigma^{m}\right)$, then

$$
[x]_{A} \mapsto[h(x)]_{B}
$$

is a Lipschitz-continuous map from $[X]_{A}$ to $[Y]_{B}$ equipped with the metric $D_{1}$. In particular, if $h$ is a conjugacy, then (6.4) is a Lipschitz-continuous homeomorphism with a Lipschitzcontinuous inverse.

Proof Choose $\delta>0$ small enough such that for all $y, y^{\prime} \in Y$ we have that if there is $s \in\{-m / 2, \ldots, m / 2\}$ with $d\left(\sigma^{s}(y), \sigma^{s}\left(y^{\prime}\right)\right) \geq 1$, then $d\left(y, y^{\prime}\right) \geq \delta$. Observe that

$$
\begin{aligned}
& \bar{D}\left(\Delta\left(\sigma^{m}, \delta, y, y^{\prime}\right)\right)=\varlimsup_{\ell \rightarrow \infty} 1 / \ell \cdot \#\left\{0 \leq k \leq \ell-1: d\left(\sigma^{k m}(y), \sigma^{k m}\left(y^{\prime}\right)\right) \geq \delta\right\} \\
& \geq \varlimsup_{\ell \rightarrow \infty} 1 / \ell \cdot \#\left\{0 \leq k \leq \ell-1: \exists s \in\{-m / 2, \ldots, m / 2\} \text { s.t. } d\left(\sigma^{k m+s}(y), \sigma^{k m+s}\left(y^{\prime}\right)\right) \geq 1\right\}
\end{aligned}
$$

which yields

$$
\bar{D}\left(\Delta\left(\sigma^{m}, \delta, y, y^{\prime}\right)\right) \geq \bar{D}\left(\Delta\left(\sigma, 1, y, y^{\prime}\right)\right)=D_{1}\left(y, y^{\prime}\right) .
$$

Next, because $h$ is uniformly continuous, there is $\eta \in(0,1]$ such that for all $x, x^{\prime} \in X$ we have that $d\left(h(x), h\left(x^{\prime}\right)\right) \geq \delta$ implies $d\left(x, x^{\prime}\right) \geq \eta$. Therefore,

$$
\begin{aligned}
\bar{D} & \left(\Delta\left(\sigma^{m}, \delta, h(x), h\left(x^{\prime}\right)\right)\right) \\
& =\varlimsup_{\ell \rightarrow \infty} 1 / \ell \cdot \#\left\{0 \leq k \leq \ell-1: d\left(\sigma^{k m}(h(x)), \sigma^{k m}\left(h\left(x^{\prime}\right)\right)\right) \geq \delta\right\} \\
& =\varlimsup_{\ell \rightarrow \infty} 1 / \ell \cdot \#\left\{0 \leq k \leq \ell-1: d\left(h\left(\sigma^{k n}(x)\right), h\left(\sigma^{k n}\left(x^{\prime}\right)\right)\right) \geq \delta\right\} \\
& \leq \varlimsup_{\ell \rightarrow \infty} 1 / \ell \cdot \#\left\{0 \leq k \leq \ell-1: d\left(\sigma^{k n}(x), \sigma^{k n}\left(x^{\prime}\right)\right) \geq \eta\right\}=\bar{D}\left(\Delta\left(\sigma^{n}, \eta, x, x^{\prime}\right)\right) .
\end{aligned}
$$

Finally, choose $\eta^{\prime}$ small enough such that for all $x, x^{\prime} \in X$ we have that $d\left(x, x^{\prime}\right) \geq \eta$ implies $d\left(\sigma^{s}(x), \sigma^{s}\left(x^{\prime}\right)\right) \geq \eta^{\prime}$ for all $s \in\{-n / 2, \ldots, n / 2\}$. Observe that $D_{\eta^{\prime}}\left(x, x^{\prime}\right)=$ $\bar{D}\left(\Delta\left(\sigma, \eta^{\prime}, x, x^{\prime}\right)\right) \geq \bar{D}\left(\Delta\left(\sigma^{n}, \eta, x, x^{\prime}\right)\right)$. Together with (6.5) and (6.6), this yields

$$
D_{\eta^{\prime}}\left(x, x^{\prime}\right) \geq \bar{D}\left(\Delta\left(\sigma^{n}, \eta, x, x^{\prime}\right)\right) \geq \bar{D}\left(\Delta\left(\sigma^{m}, \delta, h(x), h\left(x^{\prime}\right)\right)\right) \geq D_{1}\left(h(x), h\left(x^{\prime}\right)\right) .
$$

This proves that the map $[x]_{A} \mapsto[h(x)]_{B}$ is well defined and Lipschitz-continuous as a map from $\left([X]_{A}, D_{\eta^{\prime}}\right)$ to $\left([Y]_{B}, D_{1}\right)$. Using Lemma 6.2, the statement follows.

By means of Theorem 6.1(i) and Theorem 6.5 the previous statement immediately yields the following fact. 
Corollary 6.7 If $(X, \sigma)$ and $(Y, \sigma)$ are conjugate, then

$$
\underline{\operatorname{ac}}\left(\left.\sigma\right|_{X}\right)=\underline{\operatorname{ac}}\left(\left.\sigma\right|_{Y}\right) \text { and } \overline{\operatorname{ac}}\left(\left.\sigma\right|_{X}\right)=\overline{\operatorname{ac}}\left(\left.\sigma\right|_{Y}\right) \text {. }
$$

Let us point out that, given a constant length substitution $\vartheta: A \rightarrow A^{+}$, we may also consider it a map on $[\Sigma]$ by putting $\vartheta([x])=[\vartheta(x)]$. Note that for all $[x],[y] \in[\Sigma]$

$$
D_{1}(\vartheta([x]), \vartheta([y])) \leq D_{1}([x],[y]) .
$$

In a similar way, we can consider the left shift a mapping on the Besicovitch space by setting $\sigma([x])=[\sigma(x)]$. Observe that the shift becomes an isometry on $[\Sigma]$.

Proposition 6.8 A subshift $(X, \sigma)$ is mean equicontinuous if and only if the map $\left.[\cdot]\right|_{X}$ is continuous. In this case, $([X], \sigma)$ is the maximal equicontinuous factor of $(X, \sigma)$.

Proof For the first part, recall that $(X, \sigma)$ is mean equicontinuous if and only if $D_{B}$ is continuous. It hence suffices to show that $D_{B}$ and $D_{1}$ induce the same topology, that is, for all $\left(x_{n}\right)_{n \in \mathbb{N}} \in X^{\mathbb{N}}$ and $x \in X$ we have

$$
D_{1}\left(x_{n}, x\right) \stackrel{n \rightarrow \infty}{\longrightarrow} 0 \quad \text { if and only if } \quad D_{B}\left(x_{n}, x\right) \stackrel{n \rightarrow \infty}{\longrightarrow} 0 .
$$

To that end, recall that

$$
D_{1}\left(x_{n}, x\right)=\bar{D}\left(\Delta\left(\left.\sigma\right|_{X}, 1, x_{n}, x\right)\right) \leq D_{B}\left(x_{n}, x\right),
$$

see (4.1). This shows the "if"-part of (6.7). To see the "only if", suppose $D_{1}\left(x_{n}, x\right) \stackrel{n \rightarrow \infty}{\longrightarrow} 0$. Let $\varepsilon>0$ and choose $n_{\varepsilon} \in \mathbb{N}$ such that $D_{\varepsilon}\left(x_{n}, x\right)<\varepsilon$ for $n>n_{\varepsilon}$. Note that this is possible since $D_{\varepsilon}$ induces the same topology as $D_{1}$. Then we obtain for all $n>n_{\varepsilon}$ that

$$
D_{B}\left(x_{n}, x\right) \leq D_{\varepsilon}\left(x_{n}, x\right)+\varepsilon \cdot\left(1-D_{\varepsilon}\left(x_{n}, x\right)\right) \leq 2 \varepsilon .
$$

Since $\varepsilon>0$ was arbitrary, this shows $D_{B}\left(x_{n}, x\right) \stackrel{n \rightarrow \infty}{\longrightarrow} 0$ and hence (6.7).

Concerning the second part, observe that the first part and the discussion before the statement show that $[\cdot]$ is actually a factor map from $(X, \sigma)$ onto $([X], \sigma)$. Furthermore, we have that $[x]=[y]$ if and only if $D_{B}(x, y)=0$. Now, if $q$ denotes the factor map of the mean equicontinuous system $(X, \sigma)$ onto its maximal equicontinuous factor, it is well known that $q(x)=q(y)$ if and only if $D_{B}(x, y)=0$ (see for example [1, Theorem 6] or [19, Proposition 49]). Hence, [.] and $q$ identify the same points. This proves the statement.

Now, let us recall the fact that the box dimension is always bounded from below by the topological dimension (see for instance [11, Section 3.1]). With this in mind, the previous proposition has an immediate consequence: it implies that the amorphic complexity of a mean equicontinuous subshift is bounded from below by the topological dimension of its maximal equicontinuous factor.

This observation may be of particular interest for substitutive subshifts corresponding to Pisot type substitutions over two symbols (for a definition of substitutions of Pisot type, see for example Section 1.2.5 in [13]). According to [7], all substitutions of Pisot type are primitive. Moreover, all subshifts over two symbols associated to these substitutions have discrete spectrum, see [2] and [21]. Hence, we can use Corollary 5.2 to deduce that the corresponding subshifts are mean equicontinuous so that we can apply the above observation.

Two well-known examples belonging to the class of binary substitutions of Pisot type are the Fibonacci and Tribonacci substitution. For these two substitutions we obtain the concrete lower bound for the amorphic complexity of one and two, respectively. This holds true because the maximal equicontinuous factor of the former is the circle and that of the latter is the two-torus (see, again, [13] for more information). 


\section{Amorphic complexity of constant length substitution subshifts}

From now on, $\vartheta: A \rightarrow A^{+}$will always denote a primitive constant length substitution. Further, we set $D=D_{1}$. Recall that to each $\vartheta$ we can associate a minimal and uniquely ergodic subshift, denoted by $\left(X_{\vartheta}, \sigma\right)$. The goal of this section is to examine the amorphic complexity of $\left(X_{\vartheta}, \sigma\right)$ by studying its projection in the Besicovitch space $([\Sigma], D)$.

\subsection{Iterated function systems and cyclic partitions}

A finite family of contracting maps $\left(\varphi_{i}\right)_{i \in\{0, \ldots, \ell-1\}}$ on a complete metric space $(M, \rho)$ is referred to as an iterated function system (IFS). Given such an IFS, it is well known that there exists a unique compact subset $K \subseteq M$, called the attractor of the IFS, fulfilling

$$
K=\bigcup_{i=0}^{\ell-1} \varphi_{i}(K) .
$$

If, additionally, this union is disjoint, we say the IFS satisfies the strong separation condition (SSC). For more information on IFS's, see for instance [24,33].

In the following, we construct suitable iterated function systems that we will use to estimate the amorphic complexity of infinite subshifts with discrete spectrum associated to constant length substitutions. A key ingredient for doing this will be the notion of cyclic $\sigma^{n}$-minimal partitions.

Let $(X, f)$ be a minimal dynamical system. We say a partition of $X$ is cyclic if it partitions $X$ into disjoint subsets $X_{0}, \ldots, X_{m-1} \subseteq X, m \in \mathbb{N}$ with $f\left(X_{i-1}\right)=X_{i}$ for $1 \leq i<m$ and $f\left(X_{m-1}\right)=X_{0}$. A cyclic partition is called $f^{n}$-minimal if each of its elements is $f^{n}$ minimal. Note that such a partition is unique (up to cyclic permutation of its members). Given an $f^{n}$-minimal set $X_{0} \subseteq X$, it is easy to see that there is $1 \leq m \leq n$ such that $X_{0}, f\left(X_{0}\right), \ldots, f^{m-1}\left(X_{0}\right)$ is a (possibly trivial) cyclic $f^{n}$-minimal partition of $X$, see [26, Lemma 15]. For each $n \in \mathbb{N}$ we denote by $\gamma(n)$ the number of elements of a cyclic $f^{n}$ minimal partition of $X$.

Now, coming back to substitutive subshifts, observe that

$$
\vartheta \circ \sigma=\sigma^{|\vartheta|} \circ \vartheta
$$

In other words, $\vartheta$ is a factor map from $\left(X_{\vartheta}, \sigma\right)$ to $\left(\vartheta\left(X_{\vartheta}\right), \sigma^{|\vartheta|}\right)$. Hence, the minimality of $\left(X_{\vartheta}, \sigma\right)$ implies that $\vartheta\left(X_{\vartheta}\right)$ is $\sigma^{|\vartheta|}$-minimal. Since each word that appears in $\vartheta\left(X_{\vartheta}\right)$ also appears in $X_{\vartheta}$, we clearly have $\vartheta\left(X_{\vartheta}\right) \subseteq X_{\vartheta}$, see also [26, Lemma 3]. Further, if $\left(X_{\vartheta}, \sigma\right)$ is infinite, it turns out that $\gamma(|\vartheta|)=|\vartheta|$, see [8, Lemma 7]. Hence, in this case, we obtain the cyclic $\sigma^{|\vartheta|}$-minimal partition

$$
X_{\vartheta}=\bigsqcup_{i=0}^{|\vartheta|-1}\left(\sigma^{i} \circ \vartheta\right)\left(X_{\vartheta}\right) .
$$

Our goal is to carry over (7.2) to the Besicovitch space and to provide criteria which ensure that the maps $\left\{\sigma^{i} \circ \vartheta\right\}_{i \in\{0, \ldots,|\vartheta|-1\}}$ are contractions on $([\Sigma], D)$. To that end, we need the following auxiliary statement.

Proposition 7.1 Let $(X, \sigma)$ and $(Y, \sigma)$ be two minimal subshifts of $(\Sigma, \sigma)$. If $[X] \cap[Y] \neq \emptyset$, then we have that $X=Y$. 
Proof Assume for a contradiction that $X \neq Y$. Then there exists $n \in \mathbb{N}$ such that $\mathcal{L}^{n}(X) \cap$ $\mathcal{L}^{n}(Y)=\emptyset$ (see Sect. 2). Hence, for all $x \in X$ and $y \in Y$ and all $\ell \in \mathbb{N}$ we obtain

$$
1 /(\ell n) \cdot \#\left\{0 \leq k \leq \ell n-1: x_{k} \neq y_{k}\right\} \geq 1 / n,
$$

so that $D(x, y) \geq 1 / n$ which contradicts the assumptions.

Lemma 7.2 Suppose $(X, \sigma)$ is a subshift and $X_{0}, \ldots, X_{m-1} \subseteq X$ is a cyclic $\sigma^{n}$-minimal partition of $X$ for some $m, n \in \mathbb{N}$. Then

$$
[X]=\bigsqcup_{i=0}^{m-1}\left[X_{i}\right]=\bigsqcup_{i=0}^{m-1} \sigma^{i}\left(\left[X_{0}\right]\right)
$$

Proof Let $q \Sigma \rightarrow \Sigma^{\prime}=\left(A^{n}\right)^{\mathbb{Z}}$ denote the canonical higher power code of $\Sigma$, see for example Section 1.4 in [29]. Observe that $q$ is a conjugacy between $\left(\Sigma, \sigma^{n}\right)$ and $\left(\Sigma^{\prime}, \sigma\right)$. Hence, $(q(X), \sigma)$ is a subshift of $\left(\Sigma^{\prime}, \sigma\right)$ which is conjugate to $\left(X, \sigma^{n}\right)$.

Moreover, we have that $\left(q\left(X_{i}\right), \sigma\right)(i=0, \ldots, m-1)$ is minimal, so that Proposition 7.1 implies $\left[q\left(X_{i}\right)\right]_{A^{n}} \cap\left[q\left(X_{j}\right)\right]_{A^{n}}=\emptyset$ if $i \neq j$.

Finally, according to Lemma $6.6, q$ can be considered a homeomorphism from $[X]_{A}$ to $[q(X)]_{A^{n}}$ with $q\left([x]_{A}\right)=[q(x)]_{A^{n}}$ for $x \in X$. Therefore,

$$
q\left(\left[X_{i}\right]_{A} \cap\left[X_{j}\right]_{A}\right)=q\left(\left[X_{i}\right]_{A}\right) \cap q\left(\left[X_{j}\right]_{A}\right)=\left[q\left(X_{i}\right)\right]_{A^{n}} \cap\left[q\left(X_{j}\right)\right]_{A^{n}}=\emptyset,
$$

whenever $i \neq j$. Hence, $\left[X_{i}\right]_{A} \cap\left[X_{j}\right]_{A}=\emptyset$ which shows the first equality in (7.3).

For the second equality, note that $X_{i}=\sigma^{i}\left(X_{0}\right)$, since $X_{0}, \ldots, X_{m-1}$ is cyclic. The statement follows, since $\left[\sigma^{i}\left(X_{0}\right)\right]=\sigma^{i}\left(\left[X_{0}\right]\right)$.

Next, we provide a criterion to ensure that the maps $\left\{\sigma^{i} \circ \vartheta\right\}_{i \in\{0, \ldots,|\vartheta|-1\}}$ are indeed contractions. To that end, set

$$
c_{a, b}(\vartheta)=\#\left\{0 \leq j \leq|\vartheta|-1: \vartheta(a)_{j}=\vartheta(b)_{j}\right\},
$$

where $a \neq b \in A$ and define

$$
c(\vartheta)=\min _{a \neq b} c_{a, b}(\vartheta) \quad \text { and } \quad C(\vartheta)=\max _{a \neq b} c_{a, b}(\vartheta) .
$$

Note that $0 \leq c(\vartheta) \leq C(\vartheta) \leq|\vartheta|$. If there is no risk of ambiguity, we may omit the argument $\vartheta$ in the following.

Proposition 7.3 Let $[x],[y] \in[\Sigma]$. Then

$$
\frac{|\vartheta|-C}{|\vartheta|} \cdot D([x],[y]) \leq D(\vartheta([x]), \vartheta([y])) \leq \frac{|\vartheta|-c}{|\vartheta|} \cdot D([x],[y]) .
$$

Proof Given $x, y \in \Sigma$, note that

$$
\begin{aligned}
& D(\vartheta(x), \vartheta(y))=\varlimsup_{n \rightarrow \infty} \frac{1}{|\vartheta| \cdot n} \cdot \#\left\{0 \leq k \leq|\vartheta| \cdot n-1: \vartheta(x)_{k} \neq \vartheta(y)_{k}\right\} \\
& \quad=\varlimsup_{n \rightarrow \infty} \frac{1}{|\vartheta| \cdot n} \sum_{a \neq b \in A} \#\left\{0 \leq k \leq n-1:\left(x_{k}, y_{k}\right)=(a, b)\right\} \cdot\left(|\vartheta|-c_{a, b}\right)
\end{aligned}
$$




$$
\begin{aligned}
& \leq \frac{|\vartheta|-\min _{a \neq b} c_{a, b}}{|\vartheta|} \cdot \varlimsup_{n \rightarrow \infty} \frac{1}{n} \sum_{a \neq b \in A} \#\left\{0 \leq k \leq n-1\left(x_{k}, y_{k}\right)=(a, b)\right\} \\
& =\frac{|\vartheta|-c}{|\vartheta|} \cdot D(x, y) .
\end{aligned}
$$

Hence, we obtain the second inequality. The other inequality follows analogously.

Note that the estimates from the previous proposition actually hold for all $\sigma^{i} \circ \vartheta$, since the left shift $\sigma$ acts as an isometry on $([\Sigma], D)$. In particular, each of these maps is a contraction on $([\Sigma], D)$ if and only if $\vartheta$ is a contraction on $([\Sigma], D)$.

Proposition 7.4 We have that $\vartheta:[\Sigma] \rightarrow[\Sigma]$ is a contraction if and only if $c>0$.

Proof The "if"-part immediately follows from Proposition 7.3. For the other direction, suppose there are $a \neq b \in A$ with $c_{a, b}=0$. Then

$$
D((\ldots, a, a, \ldots),(\ldots, b, b, \ldots))=D(\vartheta((\ldots, a, a, \ldots)), \vartheta((\ldots, b, b, \ldots)))
$$

and the statement follows.

\subsection{Dimensional estimates and discrete spectrum}

In this section, we obtain lower and upper bounds for the amorphic complexity of $\left(X_{\vartheta}, \sigma\right)$ provided that the collection $\left\{\sigma^{i} \circ \vartheta\right\}_{i \in\{0, \ldots,|\vartheta|-1\}}$ (or some closely related collection of mappings, see Theorem 7.10) is an IFS and that $\left(X_{\vartheta}, \sigma\right)$ has discrete spectrum.

Our strategy is as follows: first, we show that the attractor of the above IFS is $\left[X_{\vartheta}\right]$ and that the IFS fulfills the strong separation condition. Then, we utilize general estimates for the box dimension of an IFS attractor (and apply Theorem 6.5). We would like to recall that the assumption of discrete spectrum is natural: if the spectrum of $\left(X_{\vartheta}, \sigma\right)$ is not purely discrete, then its amorphic complexity is infinite (see Corollary 5.2).

Theorem 7.5 Suppose that $\left(X_{\vartheta}, \sigma\right)$ is infinite, has discrete spectrum and that $c>0$. Then $\left\{\sigma^{i} \circ \vartheta\right\}_{i \in\{0, \ldots,|\vartheta|-1\}}$ is an IFS which satisfies the SSC and whose attractor is $\left[X_{\vartheta}\right]$.

Proof First, Proposition 7.4 yields that $\left\{\sigma^{i} \circ \vartheta\right\}_{i \in\{0, \ldots,|\vartheta|-1\}}$ is an IFS since $c>0$. Second, due to Corollary 5.2, $\left(X_{\vartheta}, \sigma\right)$ is mean equicontinuous. Hence, Proposition 6.8 gives that the map $X_{\vartheta} \ni x \mapsto[x]$ is continuous so that its image $\left[X_{\vartheta}\right]$ is compact.

Third, consider the cyclic $\sigma^{|\vartheta|}$-minimal partition from (7.2). By Lemma 7.2, we have

$$
\left[X_{\vartheta}\right]=\bigsqcup_{i=0}^{|\vartheta|-1} \sigma^{i}\left(\left[\vartheta\left(X_{\vartheta}\right)\right]\right)=\bigsqcup_{i=0}^{|\vartheta|-1}\left(\sigma^{i} \circ \vartheta\right)\left(\left[X_{\vartheta}\right]\right),
$$

where we used that $\vartheta$ commutes with [.] (see the discussion before Proposition 6.8).

Altogether, (7.4) shows that the attractor of $\left\{\sigma^{i} \circ \vartheta\right\}_{i \in\{0, \ldots,|\vartheta|-1\}}$ is $\left[X_{\vartheta}\right]$ and that the iterated function system fulfills the SSC.

For the next theorem recall that $0 \leq c \leq C \leq|\vartheta|$ and note that if $c=|\vartheta|$, then the substitution subshift $\left(X_{\vartheta}, \sigma\right)$ is finite. This is true because in this case the substitution $\vartheta$ has a unique fixed point given by $(\ldots, \vartheta(a), \vartheta(a), \ldots)$ with $a \in A$ arbitrary. 
Theorem 7.6 Suppose $\left(X_{\vartheta}, \sigma\right)$ is infinite, has discrete spectrum and $c>0$. We have

$$
\overline{\operatorname{ac}}\left(\left.\sigma\right|_{X_{\vartheta}}\right) \leq \frac{\log |\vartheta|}{\log |\vartheta|-\log (|\vartheta|-c)} .
$$

Furthermore, if $C \neq|\vartheta|$, then

$$
\underline{\operatorname{ac}}\left(\left.\sigma\right|_{X_{\vartheta}}\right) \geq \frac{\log |\vartheta|}{\log |\vartheta|-\log (|\vartheta|-C)} .
$$

In the proof of Theorem 7.6, we make use of the following statement. ${ }^{4}$

Proposition 7.7 ([33, Proposition 4.10]) Let $\left(\varphi_{i}\right)_{i \in\{0, \ldots, \ell-1\}}$ be an IFS on a complete metric space $(M, \rho)$ which fulfills the SSC and

$$
\rho\left(\varphi_{i}(x), \varphi_{i}(y)\right) \geq s \cdot \rho(x, y) \quad(x, y \in M, i \in\{0, \ldots, \ell-1\}),
$$

for some $s \in(0,1)$. Suppose $K \subseteq M$ is the attractor of the IFS. Then

$$
\underline{\operatorname{dim}}_{B}(K) \geq \log \ell /(-\log s) .
$$

Proof of Theorem 7.6 By means of Theorem 6.5, we obtain bounds on the upper and lower amorphic complexity of $\left(X_{\vartheta}, \sigma\right)$, by providing bounds on the upper and lower box dimension of $\left[X_{\vartheta}\right]$. Due to Theorem 7.5, $\left[X_{\vartheta}\right]$ is the attractor of the IFS $\left\{\sigma^{i} \circ \vartheta\right\}_{i \in\{0, \ldots,|\vartheta|-1\}}$. For that reason, we will obtain the above bounds as a result of the application of general estimates on the box dimension of attractors of IFS's.

To that end, let us discuss a simple (and standard) technique to obtain an upper bound for the box dimension of an attractor $K$ of an IFS $\left(\varphi_{i}\right)_{i \in\{0, \ldots, \ell-1\}}$ on a general complete metric space $(M, \rho)$. Let $r \in(0,1)$ denote a common contraction rate for $\varphi_{0}, \ldots, \varphi_{\ell-1}$. Observe that a successive application of (7.1) yields that for all $n \in \mathbb{N}$ we have

$$
K=\bigcup_{\left(i_{0}, \ldots, i_{n-1}\right) \in\{0, \ldots, \ell-1\}^{n}} \varphi_{i_{0}} \circ \ldots \circ \varphi_{i_{n-1}}(K) .
$$

Clearly, $\operatorname{diam}\left(\varphi_{i_{0}} \circ \ldots \circ \varphi_{i_{n-1}}(K)\right) \leq r^{n} \cdot \operatorname{diam}(K)$, where $\operatorname{diam}(E)$ denotes the diameter of a set $E \subseteq M$. Hence, (7.6) implies that for each $n \in \mathbb{N}$ the attractor $K$ can be covered by $\ell^{n}$ sets of diameter less or equal $r^{n} \cdot \operatorname{diam}(K)$. This yields $\overline{\operatorname{dim}}_{B}(K) \leq \log \ell /(-\log r)$.

Under the assumptions (and using the same notation) of Proposition 7.7, we altogether have the following general bounds

$$
\overline{\operatorname{dim}}_{B}(K) \leq \log \ell /(-\log r) \quad \text { and } \quad \underline{\operatorname{dim}}_{B}(K) \geq \log \ell /(-\log s) .
$$

Now, for our iterated function system $\left\{\sigma^{i} \circ \vartheta\right\}_{i \in\{0, \ldots,|\vartheta|-1\}}$ we have $\ell=|\vartheta|$. Further, due to Proposition 7.3, we get $r=(|\vartheta|-c) /|\vartheta|$ and $s=(|\vartheta|-C) /|\vartheta|$. The statement follows from (7.7).

We want to close this section with an application of Theorem 7.6 in the case of a binary alphabet. Beforehand, we will relate its assumptions to well-established properties of (constant length) substitutions and restate it accordingly, see Theorem 7.10.

\footnotetext{
4 Let us remark that, under the assumptions of Proposition 7.7 the statement in [33] actually yields an estimate $\operatorname{dim}_{H}(K) \geq \log \ell /(-\log s)$, where $\operatorname{dim}_{H}(K)$ denotes the Hausdorff dimension of the attractor $K$. However, the Hausdorff dimension is always a lower bound for the box dimension. For the definition of $\operatorname{dim}_{H}$ and its relation to $\operatorname{dim}_{B}$, see for instance [30].
} 
Let us first define the height of $\vartheta$ (see also [8, Lemma 10]) as

$$
h(\vartheta)=\max \{n \in \mathbb{N}: n \text { and }|\vartheta| \text { are coprime and } \gamma(n)=n\} .
$$

We call $\vartheta$ pure if $h(\vartheta)=1$. Note that if the subshifts associated to two primitive constant length substitutions of the same length are conjugate, then their heights agree. We say the substitution $\vartheta$ admits a coincidence (of order $k$ ) if there exist $k \in \mathbb{N}$ and $0 \leq j<|\vartheta|^{k}$ such that $\#\left\{\vartheta^{k}(a)_{j}: a \in A\right\}=1$.

Lemma 7.8 The map $\vartheta^{\ell}:[\Sigma] \rightarrow[\Sigma]$ is a contraction for some $\ell \in \mathbb{N}$ (i.e., $c\left(\vartheta^{\ell}\right)>0$ ) if and only if the substitution $\vartheta$ has a coincidence.

Proof Both directions are direct consequences of Proposition 7.4. We just show the "only if"-part. To that end, assume for a contradiction that $\vartheta^{\ell}$ is not a contraction for any $\ell \in \mathbb{N}$. Due to Proposition 7.4, this implies

$$
\forall \ell \in \mathbb{N} \exists a \neq b c_{a, b}\left(\vartheta^{\ell}\right)=0
$$

so that $\vartheta$ does not have a coincidence.

In view of the previous statement, the next assertion emphasizes that the IFS approach is tailor-made for constant length substitution subshifts with discrete spectrum.

Theorem 7.9 ([8, Theorems 4 and 7]) Suppose $\vartheta$ is pure. Then $\vartheta$ has a coincidence if and only if $\left(X_{\vartheta}, \sigma\right)$ has discrete spectrum.

Now, a substitution $\vartheta$ is said to be one-to-one if it is one-to-one as a mapping from $A$ to $A^{+}$, that is, $\vartheta(a) \neq \vartheta(b)$ whenever $a \neq b \in A$. Note that if $\vartheta$ is one-to-one, then $C \neq|\vartheta|$ and $\vartheta^{k}$ is also one-to-one for each $k \in \mathbb{N}$.

Theorem 7.10 Assume that $\vartheta$ is one-to-one and has a coincidence of order $k$ as well as that $\left(X_{\vartheta}, \sigma\right)$ is infinite. Setting $\Theta=\vartheta^{k}$, we have

$$
1 \leq \frac{\log |\Theta|}{\log |\Theta|-\log (|\Theta|-C(\Theta))} \leq \underline{\operatorname{ac}}\left(\left.\sigma\right|_{X_{\vartheta}}\right) \leq \overline{\operatorname{ac}}\left(\left.\sigma\right|_{X_{\vartheta}}\right) \leq \frac{\log |\Theta|}{\log |\Theta|-\log (|\Theta|-c(\Theta))}<\infty .
$$

Proof First, observe that $\Theta:[\Sigma] \rightarrow[\Sigma]$ is a contraction, since it has a coincide, that is, $0<c(\Theta)$. Further, $C(\Theta)<|\Theta|$ because $\Theta$ inherits the injectivity of $\vartheta$. Moreover, the substitution $\Theta$ is clearly primitive. By means of Theorem 7.6, we obtain the above bounds for the lower and upper amorphic complexity of $\left.\sigma\right|_{X_{\Theta}}$. Now, since periodic points of $\vartheta$ are also periodic points of $\Theta$, we have $X_{\vartheta}=X_{\Theta}$. The statement follows.

If $\vartheta$ is not one-to-one, we can make use of the following statement by Blanchard, Durand and Maass.

Lemma 7.11 ([6, Section 2.2]) Suppose $\vartheta$ is not one-to-one and $\left(X_{\vartheta}, \sigma\right)$ is infinite. Then there exists a primitive constant length substitution $\vartheta^{\prime}: A^{\prime} \rightarrow\left(A^{\prime}\right)^{+}$such that $\vartheta^{\prime}$ is one-toone, $\left|\vartheta^{\prime}\right|=|\vartheta|$ and $\left(X_{\vartheta^{\prime}}, \sigma\right)$ is conjugate to $\left(X_{\vartheta}, \sigma\right)$. In particular, in case that $\vartheta$ is pure, then $\vartheta^{\prime}$ is pure as well.

In the situation of the previous statement, Corollary 6.7 yields that

$$
\underline{\mathrm{ac}}\left(\left.\sigma\right|_{X_{\vartheta}}\right)=\underline{\mathrm{ac}}\left(\left.\sigma\right|_{X_{\vartheta^{\prime}}}\right) \text { and } \overline{\mathrm{ac}}\left(\left.\sigma\right|_{X_{\vartheta}}\right)=\overline{\mathrm{ac}}\left(\left.\sigma\right|_{X_{\vartheta^{\prime}}}\right) \text {. }
$$

In principle, this allows us to compute the amorphic complexity by the above methods even if $\vartheta$ is not one-to-one. We make use of this fact in the next section.

Finally, we take a look at substitutions over two symbols and establish a closed formula for the amorphic complexity of the associated subshifts. 
Corollary 7.12 Let $\vartheta:\{0,1\} \rightarrow\{0,1\}^{+}$. Suppose $\left(X_{\vartheta}, \sigma\right)$ is infinite and has discrete spectrum. Then

$$
\operatorname{ac}\left(\left.\sigma\right|_{X_{\vartheta}}\right)=\frac{\log |\vartheta|}{\log |\vartheta|-\log |\vartheta|_{*}},
$$

where $|\vartheta|_{*} \in(0,|\vartheta|)$ denotes the number of positions where $\vartheta(0)$ and $\vartheta(1)$ differ.

Proof First, observe that $c=C=|\vartheta|-|\vartheta|_{*}$. Since $\left(X_{\vartheta}, \sigma\right)$ is infinite, $\vartheta$ is one-to-one and $|\vartheta|_{*}>0$ (see the short discussion before Theorem 7.6). Furthermore, we claim that $\vartheta$ is pure. This holds because $1 \leq h(\vartheta) \leq \# A=2$ and since $h(\vartheta)=\# A$ implies that $\left(X_{\vartheta}, \sigma\right)$ is finite, see $[8$, Remark 9(i) \& (iii)]. Therefore, $\vartheta$ has a coincidence, according to Theorem 7.9. Note that this coincidence must be of order 1 and hence, $|\vartheta|_{*}<|\vartheta|$. Finally, we obtain the desired formula for the amorphic complexity from the general estimates provided in Theorem 7.10.

\subsection{Finiteness and positivity of amorphic complexity}

We finally turn to general constant length substitutions whose associated subshifts have discrete spectrum. It is an interesting observation that Corollary 7.12 implies that the amorphic complexity of infinite subshifts with discrete spectrum associated to binary substitutions is always finite and bounded from below by one. It turns out that this fact holds true in general.

Theorem 7.13 Suppose $\left(X_{\vartheta}, \sigma\right)$ is infinite and has discrete spectrum. Then

$$
1 \leq \underline{\operatorname{ac}}\left(\left.\sigma\right|_{X_{\vartheta}}\right) \leq \overline{\operatorname{ac}}\left(\left.\sigma\right|_{X_{\vartheta}}\right)<\infty \text {. }
$$

We will need the following lemma to prove Theorem 7.13.

Lemma 7.14 ([8, Lemma $17 \& 19]$ ) Assume that $\vartheta: A \rightarrow A^{+}$is not pure. Then there exists a $\sigma^{h(\vartheta)}$-minimal subset $X_{0} \subset X_{\vartheta}$ and a pure primitive constant length substitution $\eta: B \rightarrow B^{+}$such that $\left(X_{\eta}, \sigma\right)$ is conjugate to $\left(X_{0}, \sigma^{h(\vartheta)}\right)$.

The substitution $\eta$ from Lemma 7.14 is also called the pure base of $\vartheta$.

Proof of Theorem 7.13 First, suppose $\vartheta$ is pure. W.l.o.g. we may assume that $\vartheta$ is one-to-one because of Lemma 7.11 and (7.8). Further, due to Theorem 7.9, $\vartheta$ has a coincidence of order $k \in \mathbb{N}$. By Theorem 7.10, we get $\underline{\operatorname{ac}}\left(\left.\sigma\right|_{X_{\vartheta}}\right) \geq 1$ and $\overline{\operatorname{ac}}\left(\left.\sigma\right|_{X_{\vartheta}}\right)<\infty$.

Now, assume that $\vartheta$ is not pure. Consider the $\sigma^{h(\vartheta)}$-minimal subset $X_{0} \subset X_{\vartheta}$ provided by Lemma 7.14. By definition of $h(\vartheta)$, we get that $X_{0}, \sigma\left(X_{0}\right), \ldots, \sigma^{h(\vartheta)-1}\left(X_{0}\right)$ is a cyclic $\sigma^{h(\vartheta)}$-minimal partition of $X_{\vartheta}$. Therefore, Lemma 7.2 yields

$$
\left[X_{\vartheta}\right]=\bigsqcup_{i=0}^{h(\vartheta)-1} \sigma^{i}\left(\left[X_{0}\right]\right) .
$$

Further, because $\sigma$ is an isometry on $([\Sigma], D)$, we can use Theorem 6.1(ii) to obtain

$$
\underline{\operatorname{dim}}_{B}\left(\left[X_{0}\right]\right)=\underline{\operatorname{dim}}_{B}\left(\left[X_{\vartheta}\right]\right) \leq \overline{\operatorname{dim}}_{B}\left(\left[X_{\vartheta}\right]\right)=\overline{\operatorname{dim}}_{B}\left(\left[X_{0}\right]\right) .
$$

Finally, since $\left(X_{\eta}, \sigma\right)$ is conjugate to $\left(X_{0}, \sigma^{h(\vartheta)}\right)$, Lemma 6.6 yields a Lipschitzcontinuous homeomorphism from $\left(\left[X_{\eta}\right]_{B}, D\right)$ to $\left(\left[X_{0}\right]_{A}, D\right)$ with a Lipschitz-continuous inverse. By Theorem 6.1(i) and Theorem 6.5, we hence have

$$
\underline{\operatorname{ac}}\left(\left.\sigma\right|_{X_{\eta}}\right)=\underline{\operatorname{ac}}\left(\left.\sigma\right|_{X_{\vartheta}}\right) \leq \overline{\operatorname{ac}}\left(\left.\sigma\right|_{X_{\vartheta}}\right)=\overline{\operatorname{ac}}\left(\left.\sigma\right|_{X_{\eta}}\right) \text {. }
$$

Since $\eta$ is pure, the statement follows from the first part of the proof. 
Let us point out that the proof of Theorem 7.13 and its ingredients in principle yield an algorithm to compute concrete bounds for the amorphic complexity of $\left(X_{\vartheta}, \sigma\right)$ :

(1) Follow the proof of [8, Theorem 14], to determine the pure base $\eta: B \rightarrow B^{+}$of $\vartheta$ (which coincides with $\vartheta$ if $\vartheta$ is pure).

(2) If $\eta$ is not one-to-one, follow the method described in [6, Section 2.2] to obtain the one-to-one substitution $\eta^{\prime}: B^{\prime} \rightarrow\left(B^{\prime}\right)^{+}$(see Lemma 7.11). Otherwise, set $\eta^{\prime}=\eta$. Recall that in any case $\eta^{\prime}$ is pure.

(3) Apply Theorem 7.9 to find $k \in \mathbb{N}$ such that $\eta^{\prime}$ has a coincidence of order $k$. Then, Theorem 7.10 immediately yields concrete bounds for the lower and upper amorphic complexity of $\left(X_{\vartheta}, \sigma\right)$ depending on $C\left(\eta^{\prime k}\right)$ and $c\left(\eta^{\prime k}\right)$, respectively.

We would like to remark that as a corollary of our results, the amorphic complexity readily distinguishes constant length substitutions which correspond to different dynamical behavior according to the next assertion.

\section{Corollary 7.15 Suppose $\vartheta: A \rightarrow A^{+}$is a primitive substitution of constant length. Then}

(i) $\operatorname{ac}\left(\left.\sigma\right|_{X_{\vartheta}}\right)=0$ iff $\left(X_{\vartheta}, \sigma\right)$ is finite;

(ii) $1 \leq \underline{\operatorname{ac}}\left(\left.\sigma\right|_{X_{\vartheta}}\right) \leq \overline{\mathrm{ac}}\left(\left.\sigma\right|_{X_{\vartheta}}\right)<\infty$ iff $\left(X_{\vartheta}, \sigma\right)$ has discrete spectrum and is infinite;

(iii) $\operatorname{ac}\left(\left.\sigma\right|_{X_{\vartheta}}\right)=\infty$ iff $\left(X_{\vartheta}, \sigma\right)$ has partly continuous spectrum.

Proof Clearly, the amorphic complexity of finite subshifts is always zero. Further, recall that the amorphic complexity of $\left(X_{\vartheta}, \sigma\right)$ is infinite if $\left(X_{\vartheta}, \sigma\right)$ has partly continuous spectrum, according to Corollary 5.2. By Theorem 7.13, we have that if $\left(X_{\vartheta}, \sigma\right)$ has discrete spectrum and is infinite, then $1 \leq \underline{\operatorname{ac}}\left(\left.\sigma\right|_{X_{\vartheta}}\right) \leq \overline{\operatorname{ac}}\left(\left.\sigma\right|_{X_{\vartheta}}\right)<\infty$. The statement follows.

Acknowledgements Open access funding provided by University of Vienna.

Open Access This article is distributed under the terms of the Creative Commons Attribution 4.0 International License (http://creativecommons.org/licenses/by/4.0/), which permits unrestricted use, distribution, and reproduction in any medium, provided you give appropriate credit to the original author(s) and the source, provide a link to the Creative Commons license, and indicate if changes were made.

\section{References}

1. Auslander, J.: Mean-L-stable systems. Ill. J. Math. 3(4), 566-579 (1959)

2. Barge, M., Diamond, B.: Coincidence for substitutions of Pisot type. Bull. Soc. Math. France 130(4), 619-626 (2002)

3. Barreira, L., Pesin, Ya.B.: Lyapunov Exponents and Smooth Ergodic Theory, volume 23 of University Lecture Series. American Mathematical Society, (2002)

4. Billingsley, P.: Hausdorff dimension in probability theory. Ill. J. Math. 4, 187-209 (1960)

5. Blanchard, F., Formenti, E., Kůrka, P.: Cellular Automata in the Cantor, Besicovitch, and Weyl Topological Spaces. Complex Syst. 11(2), 107-123 (1997)

6. Blanchard, F., Durand, F., Maass, A.: Constant-length substitutions and countable scrambled sets. Nonlinearity 17(3), 817-833 (2004)

7. Canterini, V., Siegel, A.: Geometric representation of substitutions of Pisot type. Trans. Am. Math. Soc. 353(12), 5121-5144 (2001)

8. Dekking, F.M.: The spectrum of dynamical systems arising from substitutions of constant length. Zeitschrift für Wahrscheinlichkeitstheorie und Verwandte Gebiete 41(3), 221-239 (1978)

9. Downarowicz, T.: Survey of odometers and Toeplitz flows. In S.F. Kolyada, Y.I. Manin, and T. Ward, editors, Algebraic and Topological Dynamics, volume 385, pages 7-37. American Mathematical Society, (2005)

10. Downarowicz, T., Glasner, E.: Isomorphic extensions and applications. Topol. Methods Nonlinear Anal. 48(1), 321-338 (2016) 
11. Edgar, G.A.: Integral, probability, and fractal measures. Springer, New York (1998)

12. Fan, A., Jiang, Y.: Oscillating sequences, MMA and MMLS flows and Sarnak's conjecture. Ergod. Theory Dyn. Syst. 38(5), 1709-1744 (2018)

13. Fogg, N.P.: Substitutions in Dynamics. Arithmetics and Combinatorics. Springer-Verlag, Berlin Heidelberg (2002)

14. Fomin, S.: On dynamical systems with a purely point spectrum. Doklady Akademii Nauk SSSR 77, 29-32 (1951). In Russian

15. Fuhrmann, G., Gröger, M., Jäger, T., Kwietniak, D.: Amorphic complexity of group actions with applications to quasicrystals. In preparation

16. Fuhrmann, G., Gröger, M., Lenz, D.: The structure of mean equicontinuous group actions. Preprint arXiv:1812.10219 (2018)

17. Fuhrmann, G., Gröger, M., Jäger, T.: Amorphic complexity. Nonlinearity 29(2), 528-565 (2016)

18. Furstenberg, H.: Disjointness in ergodic theory, minimal sets, and a problem in Diophantine approximation. Math. Syst. Theory Int. J. Math. Comput. Theory 1, 1-49 (1967)

19. García-Ramos, F.: Weak forms of topological and measure-theoretical equicontinuity: relationships with discrete spectrum and sequence entropy. Ergod. Theory Dyn. Syst. 37(4), 1211-1237 (2017)

20. Gottschalk, W.H.: Substitution minimal sets. Trans. Am. Math. Soc. 109(3), 467-491 (1963)

21. Hollander, M., Solomyak, B.: Two-symbol Pisot substitutions have pure discrete spectrum. Ergod. Theory Dyn. Syst. 23(2), 533-540 (2003)

22. Host, B.: Valeurs propres des systémes dynamiques définis par des substitutions de longueur variable. Ergod. Theory Dyn. Syst. 6(4), 529-540 (1986)

23. Huang, W., Wang, Z., Zhang, G.: MÖbius disjointness for topological models of ergodic systems with discrete spectrum. J. Mod. Dyn. 14, 277-290 (2019)

24. Hutchinson, J.E.: Fractals and self similarity. Indiana Univ. Math. J. 30(5), 713-747 (1981)

25. Kůrka, P.: Topological and symbolic dynamics. Collection SMF. Société mathématique de France, Paris, (2003)

26. Kamae, T.: A topological invariant of substitution minimal sets. J. Math. Soc. Jpn 24(2), 285-306 (1972)

27. Klein, B.G.: Homomorphisms of symbolic dynamical systems. Math. Syst. Theory 6(1), 107-122 (1972)

28. Li, J., Tu, S., Ye, X.: Mean equicontinuity and mean sensitivity. Ergod. Theory Dyn. Syst. 35(8), 25872612 (2015)

29. Lind, D., Marcus, B.: An Introduction to Symbolic Dynamics and Coding. Cambridge University Press, Cambridge (1995)

30. Pesin, Ya.B.: Dimension Theory in Dynamical Systems. Chicago Lectures in Mathematics. University of Chicago Press, Chicago (1997)

31. Qiu, J., Zhao, J.: A note on mean equicontinuity. Journal of Dynamics and Differential Equations. To appear

32. Queffélec, M.: Substitution Dynamical Systems - Spectral Analysis. Lecture Notes in Mathematics, 2nd edn. Springer-Verlag, Berlin (2010)

33. Rajala, T., Vilppolainen, M.: Weakly controlled Moran constructions and iterated functions systems in metric spaces. Ill. J. Math. 55(3), 1015-1051 (2011)

34. Simpson, S.G.: Symbolic dynamics: entropy $=$ dimension $=$ complexity. Theory Comput. Syst. 56(3), 527-543 (2015)

35. Walters, P.: An Introduction To Ergodic Theory, volume 79 of Graduate Texts in Mathematics. Springer, New York (1982)

Publisher's Note Springer Nature remains neutral with regard to jurisdictional claims in published maps and institutional affiliations. 